\title{
Stochastic resonance in the presence of spatially localized structures
}

\author{
Ivan Rabbiosi* \\ Department of Physics, University of Strathclyde, 107 Rottenrow, Glasgow G4 ONG, Scotland, United Kingdom \\ and IMEDEA, Campus UIB, E-07071 Palma de Mallorca, Spain \\ Andrew J. Scroggie ${ }^{\dagger}$ and Gian-Luca Oppo ${ }^{\ddagger}$ \\ Department of Physics, University of Strathclyde, 107 Rottenrow, Glasgow G4 ONG, Scotland, United Kingdom
}

(Received 11 April 2003; published 8 September 2003)

\begin{abstract}
Stable spatially localized structures exist in a wide variety of spatially extended nonlinear systems, including nonlinear optical devices. We study stochastic resonance (SR) in models of optical parametric oscillators in the presence of a spatially uniform time-periodic driving and in a regime where two equivalent states with equal intensity but opposite phase exist. Diffraction and nonlinearity enable the existence of localized states, formed by the locking of kinks and antikinks and displaying spatially damped oscillatory tails (in one dimension) or the stabilization of dark ring cavity solitons (in two dimensions). We show that SR is inhibited at low driving amplitudes by the presence of localized states which obstruct the front motion. For larger driving amplitudes, in the regime where localized states cease to be stable, we observe instead an enhancement of SR.
\end{abstract}

DOI: 10.1103/PhysRevE.68.036602

PACS number(s): 47.52.+j, 42.65.Sf, 47.20.Ky, 02.50.Ey

The presence of noise in a nonlinear system often results in seemingly counterintuitive effects. One example is stochastic resonance (SR) which is nonetheless one of the most studied and well understood [1,2]. During recent years there has been an increasing interest in the study of stochastic effects in spatially extended nonlinear systems [3]. The introduction of spatial degrees of freedom gives rise to a new series of possible features that influence the SR phenomena with respect to the zero-dimensional case [4]. Previous studies have shown that the coupling of a certain number of resonators into an array can enhance the signal-to-noise ratio (SNR) [5-7]. This has been referred to as array enhanced SR (AESR) [8-10]. It is important to point out that the AESR is an effect due to the discretization and cannot be observed in continuous systems where coupling constants often just play the role of scaling factors. Here we study enhancements and inhibitions of SR not due to changes in the coupling constants as Refs. [8-10] but to the presence of solitary structures.

Our investigation focuses on one-dimensional (1D) and 2D models of optical parametric oscillators (OPO's), one of the prototypes for the study of pattern formation, solitons, localized structures, etc., in nonlinear optics. The OPO is a nonpotential system that has attracted a lot of attention both from the fundamental [11-14] and experimental sides [15]. A $\pi$-phase symmetry enables the existence of two equivalent stable states with opposite phase but the same intensity for the signal field. Domain wall (DW) solutions, or kinks, separating the two equivalent states are characterized by spatially damped oscillatory tails which are ubiquitous in nonlinear optics in the presence of nonlinearity and diffraction. Mutual attraction or repulsion between a pair of DW vanishes at certain characteristic distances giving rise to stable spatially

\footnotetext{
*Electronic address: ivan@phys.strath.ac.uk

${ }^{\dagger}$ Electronic address: andrew@ phys.strath.ac.uk

‡Electronic address: gianluca@phys.strath.ac.uk
}

localized states (LS's). Two-state transitions are activated by stochastic fluctuations, through the nucleation of a small domain of critical size. In the presence of a spatially isotropic time-periodic modulation, transitions between the two homogeneous states tend to synchronize with the driving and are enhanced by the driven front motion. The best synchronization is achieved for an optimal noise intensity where the signal-to-noise ratio (SNR) is maximized.

The implications that the presence of LS's have on the appearence of SR are manifold and motivate the present study. The choice of a nonvariational system [16] such as the OPO's should not be considered a limitation on our results. This model is in fact representative of an entire class of optical devices and of systems beyond nonlinear optics.

Our work is organized as follows. In Sec. I we introduce the OPO models. We initially discuss the $1 \mathrm{D}$ case in Sec. II where we study the LS stability in the presence of constant and time-dependent bias and we also discuss the implications of their stability when the system undergoes a periodic driving. We demonstrate the existence of SR for this system and we characterize the phenomenon in the presence of LS's on varying the kink density and driving amplitude. The characterization of SR is done by considering the average kink density. Finally, in Sec. III we generalize our results to $2 \mathrm{D}$ models.

\section{THE MODEL}

We consider models of a stochastically driven degenerate OPO (DOPO) in one transverse dimension and for convenience we set to zero the detunings for pump and signal field. In the doubly resonant model (DRDOPO), where both pump and the degenerate signal [17] fields are resonated in a cavity, we have the following system of stochastic partial differential equations:

$$
\partial_{t} A_{0}=\Gamma\left[-A_{0}+E-A_{1}^{2}\right]+i a \nabla^{2} A_{0},
$$




$$
\partial_{t} A_{1}=\left[-A_{1}+A_{0} A_{1}^{*}+i 2 a \nabla^{2} A_{1}\right]+\mu+\xi(\vec{x}, t),
$$

where $A_{0}$ and $A_{1}$ are two complex functions representing the pump and degenerate signal field, respectively, $\partial_{t}=\partial / \partial t$, and $\nabla^{2}=(\partial / \partial x)^{2}+(\partial / \partial y)^{2}$. On the right-hand side of Eq. (1) $\Gamma$ is the ratio between the pump and degenerate signal decay rates in the cavity, $E$ is the amplitude of the external pump field, and $a$ is the diffraction constant. The term $\mu$ represents a perturbation which will be conveniently chosen either constant or time dependent and the complex term $\xi(\vec{x}, t)$ is Gaussian white noise which satisfies $\left\langle\xi(\vec{x}, t) \xi^{*}\left(\vec{x}^{\prime}, t^{\prime}\right)\right\rangle$ $=2 \epsilon \delta\left(\vec{x}-\vec{x}^{\prime}\right) \delta\left(t-t^{\prime}\right)$, with $\epsilon$ the noise strength. For $\Gamma \gg 1$ the cavity becomes transparent to the pump field and system (1) reduces to

$$
\partial_{t} A_{1}=-A_{1}+E A_{1}^{*}+A_{1}\left|A_{1}\right|^{2}+i a \nabla^{2} A_{1}+\mu+\xi(\vec{x}, t),
$$

which is therefore called the singly resonant DOPO.

When $\mu$ and $\epsilon$ vanish, Eqs. (1) and (2) possess three homogeneous steady state solutions for the degenerate signal field $\left(A_{1}=0, \pm \sqrt{E-1}\right)$ which are present above the threshold for the degenerate signal generation. The trivial solution is unstable whereas the other two, which we call $A_{1}^{ \pm}$, are stable. DW solutions (also called kinks in 1D models) connecting the two stable equilibrium states are the simplest among the nonhomogeneous solutions. DW's are also stable and correspond to heteroclinic orbits in the phase space that start from one equilibrium state for $\vec{x} \rightarrow-\infty$, vanish at the origin, the kink core, and end on the opposite equilibrium state for $\vec{x} \rightarrow+\infty$. Since the stable homogeneous states are real, DW's manifest themselves only in the real part of $A_{1}$ where their profile is characterized in Eq. (1) by oscillatory tails that are due to the coupling of the nonlinearity with diffraction. In Eq. (2) these oscillatory tails are critically damped. Since we are interested in the switching between stable equilibrium states we focus only on the real part $u$ of $A_{1}=u+i w$.

\section{THE 1D CASE $\left(\nabla^{2}=\partial_{x x}\right)$}

In one dimension the interaction between kink and antikink vanishes at certain locking distances $s_{j}$ where the DW's lock forming stable LS's [13]. The distances $s_{j}$ also identify each $j$-order localized structure. The smallest separation $s_{0}$ corresponds to the stable spatial soliton. The stability of the locked states decreases exponentially with $s_{j}[18]$, so that the most stable is the $j=0$ solitonlike structure. We remark that all these LS's are homoclinic solutions starting and ending on the same stable homogeneous state without intercepting the other stable state and without passing through the origin of the phase space. When dealing with DW's we identify the core of the kink with the point where both real and imaginary parts vanish. This definition is then compromised for small interaction distances, where the concept of a DW as a heteroclinic solution connecting two homogeneous phases loses validity. However, in a broader sense, since $u$ is forced to vanish both for heteroclinic and homoclinic solutions, we identify the crossing coordinates $x_{k}\left[u\left(x_{k}\right)=0\right]$ with the kink cores or equivalently the center of mass of the kinks. Periodic boundary conditions allow just an even number of $x_{k}$.

Above the threshold for degenerate signal generation, multiple locked-state solutions-emerging under small random perturbation from the zero-signal unstable solution-are characterized by asymptotically stable spatial chaos [18]. Under certain conditions and in the absence of forcing ( $\mu$ $=0$ ), noise can suppress chaos inducing the growth of spatially periodic arrays of solitons [19].

\section{A. LS stability with constant or periodic bias}

We first consider the case $\epsilon=0$. The symmetry between the two equivalent stable states can be broken by the addition of a constant perturbation $\mu$ in the equation for the degenerate signal field. The sign of $\mu$ determines which of the two steady states becomes more stable, forcing kinks that are initially stable and far from each other to move. The direction of the motion is that leading to the suppression of the less stable state. If a pair of defects encloses the less (more) stable homogeneous state they are driven towards (away from) each other.

We study the stability of the $j$-order LS by applying a perturbation that tends to contract or expand the separation between the kink pair. Equations (1) and (2) are integrated numerically by using a Milshtein's method as described in Ref. [20]. For increasing values of $|\mu|$ starting from zero, $j$-order LS loses stability for $|\mu|>\alpha_{j}$ and for $|\mu|>\beta_{j}$ when subject to contraction and expansion, respectively, where $\alpha_{j}>\beta_{j}$. Since the stability of the $j$-order LS decreases with increasing order, we also have

$$
\alpha_{0}>\beta_{0}>\alpha_{1}>\beta_{1}>\cdots>\alpha_{j}>\beta_{j},
$$

with

$$
\lim _{j \rightarrow \infty} \alpha_{j}=\lim _{j \rightarrow \infty} \beta_{j}=0 .
$$

In fact, $\left\{\alpha_{j}\right\}$ and $\left\{\beta_{j}\right\}$ decay much more rapidly in the case of Eq. (2) where already $\alpha_{0}^{(2)} \ll \alpha_{0}^{(1)}$ and $\beta_{0}^{(2)} \ll \beta_{0}^{(1)}$ (here the upper indices indicate systems (1) and (2), respectively). Relation (3) is obvious in the case where a potential for the defect interaction can be deduced, as in Ref. [18]. In that case the potential barriers on either side of the potential well in which the defect sits have different heights due to the exponential decay of the interaction. Relation (3) suggests that the same character persists in a situation far from variational.

In Fig. 1 we plot the stability regions of the first two stable localized solutions of Eq. (1) and of the solitonlike solution of Eq. (2), which are shown in Figs. 1(a), 1(b), and 1(c), respectively, in the space of parameters $E$ and $\mu$. Note that for any solution $A_{1}$ there exist the negative $-A_{1}$ too and since $\alpha_{j} \neq \beta_{j}$ we obtain two different stability regions $V_{\uparrow j}$ and $V_{\downarrow j}$ (indicated by arrows in Fig. 1) for an upward (solid arrow) and a downward (dashed arrow) oriented localized solution. As a consequence a kink pair subject to contraction becomes subject to expansion if we change the sign of $\mu$ (or 


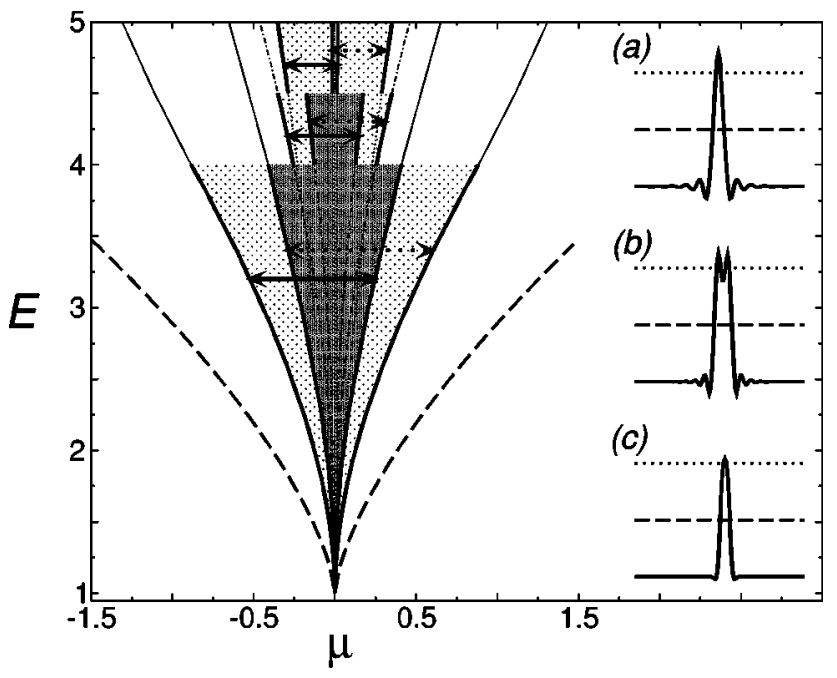

FIG. 1. Stability regions of the first two $(j=0,1)$ stable localized solutions of Eq. (1) (shaded up to $E=3$ and $E=4$, respectively) and of the $j=0$ order solution of Eq. (2) (shaded up to $E$ $=5$ ). Arrow pairs indicate the widths of the stability regions for an upward (solid arrow) and downward (dotted arrow) oriented state. Dark gray shading corresponds to values of $E$ and $\mu$ where a state and its negative are stable, while light gray shading corresponds to regions where only one of the two is stable. Insets: (a) and (b) 0 -order and 1-order solutions of Eq. (1). (b) 0-order solution of Eq. (2).

that of $\left.A_{1}\right)$. Therefore, only inside their intersection $W_{j}$ $=V_{\uparrow j} \cap V_{\downarrow j}$ (dark gray shading) are both the $j$-order state and antistate stable. Outside the inner region $W_{j}$ there is an intermediate region $V_{j}=\left(V_{\uparrow j} \cup V_{\downarrow j}\right)-W_{j}$ (light gray shading) where $j$-order locked states are stable or unstable depending on their orientation. The most external curve (dotted line in Fig. 1) is the threshold that defines the region $\Sigma$ of existence of two stable homogeneous solutions $A_{1}^{ \pm}(\mu)$, which is given by

$$
\mu_{t h}=2[(E-1) / 3]^{3 / 2}
$$

In the region $Q_{j}=\Sigma-\left(V_{j} \cup W_{j}\right)$ none of the $j$-order locked states exist. To help with the interpretation of Fig. 1, the regions of stability have been shaded up to $E=3$ and $E=4$ for the soliton and double-peak solutions of Eq. (1), respectively, and up to $E=5$ for the soliton solution of Eq. (2). Similar plots are obtained for higher order LS's.

\section{B. Driven front motion}

Since we are interested in the transitions of $u=\operatorname{Re}\left(A_{1}\right)$ between the two equilibrium states $A_{1}^{ \pm}(t)$, we map the function $u(x, t)$ into the two discrete states \pm 1 , with the kink position $x_{k}$ lying in the discontinuity. We define $U(x, t)$ $=u /|u|$ ignoring in this way the oscillations around the equilibrium states imposed by $\mu$ (the intrawell motion in the potential representation). However we point out that our results are not affected qualitatively by this filtering. We define the spatially averaged power spectrum as

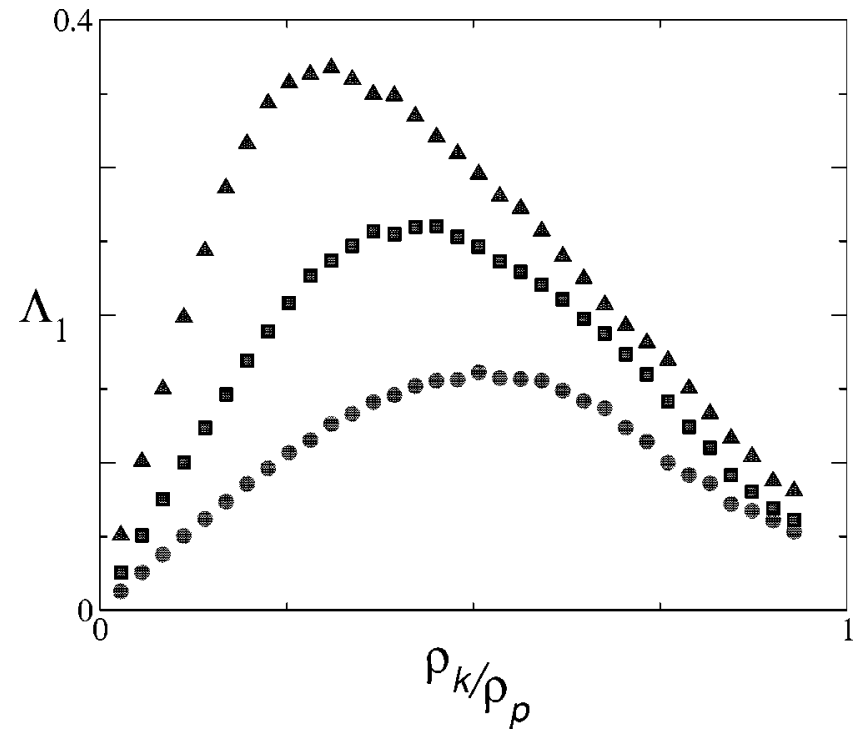

FIG. 2. Behavior of the signal power $\Lambda(\Omega)$ for Eq. (1) on varying the kink density $\rho_{k} / \rho_{p}$, where $\rho_{p}$ is the maximum allowed density of kinks: $\Omega=\pi \times 10^{-2}$ (triangles), $\Omega=5 \pi \times 10^{-3}$ (squares), and $\Omega=25 \pi \times 10^{-4}$ (circles). Each point has been obtained by averaging over 100 different initial conditions. Parameters are $\bar{\mu}=0.1, \Gamma=1, E=3$.

$$
\Lambda(\omega)=\frac{1}{L} \int_{0}^{L} d x\left|\int_{\infty}^{+\infty} U(x, t) e^{i \omega t} d t\right|^{2}
$$

We now consider $\mu(t)=\bar{\mu} \sin (\Omega t)$, with $\bar{\mu}$ constant. An isolated kink displays a periodic motion driven by $\mu$, its position being given by $x_{k}(t)=-\sigma_{k}(v / \Omega) \cos (\Omega t)$, where $\sigma_{k}=\left[d u\left(x_{k}\right) / d x\right]\left|d u\left(x_{k}\right) / d x\right|^{-1}= \pm 1$ is the crossing direction. Here $v$ is the constant velocity of the defect, which is found using singular perturbation theory [22] when $\mu$ is constant. Thus each isolated kink gives a contribution to $\Lambda$ at the frequency $\Omega$. It is interesting to note that for a given $\Omega$ and $\bar{\mu}$ there exists a kink density $\rho_{S}$ for which the signal is maximized. For instance, we consider a random distribution of $n_{k}$ kinks with density $\rho_{k}=n_{k} / L$ placed at arbitrary positions. Moreover, we choose $\bar{\mu}<\alpha_{0}$. In this way $\rho_{k}$ remains constant for an arbitrarily long time, since without noise there cannot be any production or annihilation of kinks. In Fig. 2 we plot $\Lambda(\Omega)$. Each point has been averaged over 100 random initial conditions with a fixed kink density $\rho_{k} . \Lambda(\Omega)$ is obviously zero when $\rho_{k}=0$ ( $u$ in one equilibrium state) and it increases on increasing $\rho_{k}$ reaching a maximum. However as the distance between adjacent kinks shortens on increasing $\rho_{k}$, their motion becomes more and more restricted and the signal eventually decreases (see Fig. 2). This particular resonance also depends on the angular frequency of the bias. On increasing $\Omega$ the maximum signal shifts to larger values of $\rho_{k}$ and simultaneously decreases: $\Omega=\pi \times 10^{-2}$ (triangles), $\Omega=5 \pi \times 10^{-2}$ (squares), $\Omega=25 \pi \times 10^{-2}$ (circles). We remark that this type of resonance is possible only because of the locking of DW's and because of the hierarchical 

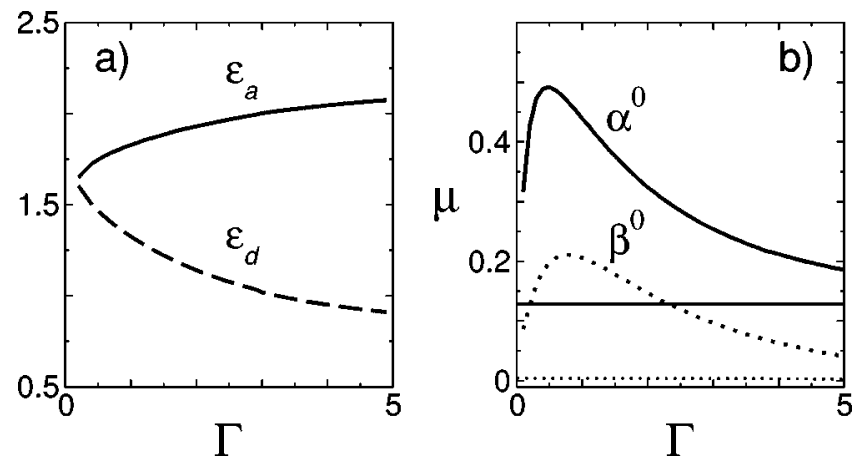

FIG. 3. (a) The threshold amplitude $\mathcal{E}_{d}$ (dashed line) and $\mathcal{E}_{a}$ (solid line). Parameters are $\Gamma=1, E=3$, and $a=0.5$. (b) Range of stability of the 0 -order LS's in Eq. (1) when subject to contraction (solid line) and expansion (dotted line) in the presence of bias $\mu$. Straight lines correspond to stability thresholds for Eq. (2).

structure of their existence limits (3). A purely attractive interaction between kinks would result in their annihilation after a suitable time.

\section{Control of stability and growth of spatial structures}

Tuning the parameter $\Gamma$ in Eq. (1) corresponds to changing the pump cavity finesse with respect to the degenerate signal finesse. We remark that this is possible by virtue of the double time-scale structure of Eq. (1) and is therefore absent in Eq. (2). The effects of this variation are manifold and we address them in order. First, from the deterministic side, we have the following facts.

(i) The profile of the LS's (both stable and unstable) changes with $\Gamma$. For example, on reducing $\Gamma$, the amplitude of the damped oscillations increases. Importantly, a change in the profile of the LS's affects the activation (deactivation) amplitude, $\mathcal{E}_{a}\left(\mathcal{E}_{d}\right)$. This is defined as the threshold that a perturbation needs to overcome in magnitude in order to generate (erase) a soliton $[19,21]$. A plot of these two quantities versus $\Gamma$ is reported in Fig. 3(a) (solid and dashed lines, respectively) versus $\Gamma$ for $E=3$.

(ii) The existence limits of the LS's in the parameter space $(\mu, E)$ are functions of $\Gamma$. In Fig. 3(b) we plot the existence range of the stable 0 -order LS's as a function of $\Gamma$ when subject to contraction (solid line) and expansion (dotted line), respectively, imposed by the bias $\mu$. For large $\Gamma$ the curves approach asymptotically the stability thresholds for the corresponding LS's of Eq. (2). These thresholds are indicated by straight lines in Fig. 3(b). Note also that, by comparing the widths of the stability regions in Fig. 1, it is immediately evident that solitons in Eq. (1) are more stable than their counterpart in Eq. (2).

(iii) The velocity of a DW when subject to a uniform external perturbation $\mu$ increases with $\Gamma$. The curves of the velocity calculated using, for example, the method described in Refs. [21,22] are displayed in Fig. 4(a) for $\Gamma=0.2$ (dashed line), $\Gamma=1$ (dotted line), and $\Gamma=5$ (dot-dashed line). The corresponding values obtained from numerical analysis are represented by symbols.
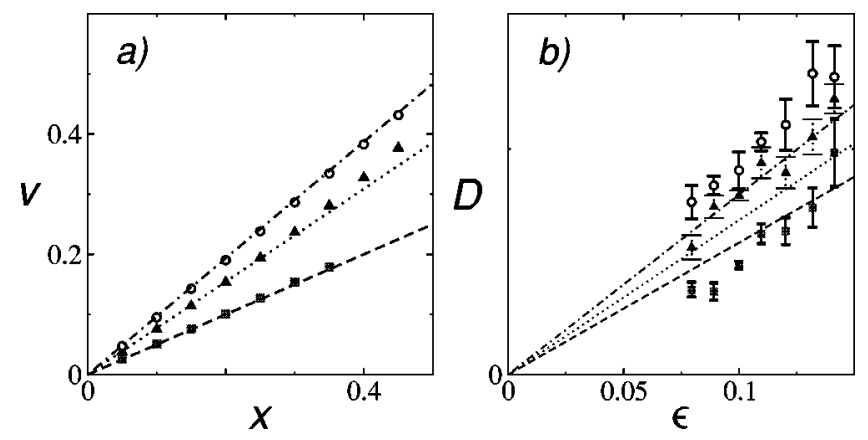

FIG. 4. (a) Kink velocity when a constant perturbation $\mu$ is externally added. Lines represent the calculated values of $v$ for $\Gamma$ $=0.2$ (dashed line), $\Gamma=1$ (dotted line), and $\Gamma=5$ (dot-dashed line), while symbols are obtained by a direct measurement of the velocity from the numerical simulations. (b) Diffusion coefficient vs the noise intensity $\epsilon$. Lines represent the predicted values for $\Gamma=0.2$ (dashed line), $\Gamma=1$ (dotted line), and $\Gamma=5$ (dot-dashed line), while symbols are the results of the statistics for the values $\Gamma=0.2$ (squares), $\Gamma=1$ (triangles), and $\Gamma=5$ (circles) obtained by a direct measurement.

Second, the stochastic dynamics of kinks is also significantly affected by variations of $\Gamma$ and we have the following facts.

(i') Since the ratio between $\mathcal{E}_{a}$ and $\mathcal{E}_{d}$ determines the equilibrium kink density, one observes larger kink densities on reducing $\Gamma$. In particular, when the activation threshold $\mathcal{E}_{a}$ becomes smaller than $\mathcal{E}_{d}, \rho(\epsilon)$ no longer goes to zero for $\epsilon \rightarrow 0$.

(ii') The kink diffusion increases with $\Gamma$. Fig. 4b shows the diffusion coefficient $D$ versus the noise intensity $\epsilon$. Lines represent the predicted values for $\Gamma=0.2$ (dashed line), $\Gamma$ $=1$ (dotted line), and $\Gamma=5$ (dot-dashed line). These are obtained by using singular perturbation theory. The values measured using a statistical method, which consists in measuring the time-dependent variance of the random walk of the kink over $10^{3}$ different simulations, are represented by the symbols: $\Gamma=0.2$ (squares), $\Gamma=1$ (triangles), and $\Gamma=5$ (circles).

A characterization of SR in the DOPO model needs to consider these five points. In particular (i), (ii), and (i') are sufficient, as we shall see, to explain most of the behavior observed [note that $\left(\mathrm{i}^{\prime}\right)$ is a consequence of (i)]. As a matter of fact, points (iii) and (ii') concern the "mobility" of isolated DW's, which is reflected in their velocity and diffusion when subject to deterministic and stochastic perturbations, respectively. The kink dynamics in the presence of other kinks strongly depends on their mutual interaction. Due to the locking of kinks for short interaction distances the front motion may temporarily freeze thus inhibiting the synchronization process. Since the average interaction distance between kinks is $1 / \rho$, the inhibition increases with $\rho$.

In Sec. II B we showed that the driven motion of kinks contributes to the synchronization mechanism for low noise intensities. We also underlined the relevance of the density of kinks for the signal power. As we shall see, the onset of the signal power amplification on increasing $\epsilon$ depends on $\Gamma$ as a consequence of $\left(i^{\prime}\right)$. The SR curve broadens on reducing $\Gamma$ 
since $\rho$ becomes significant even for very low noise intensities. Importantly, from (ii) one deduces that the behavior of SR also depends on the amplitude $\bar{\mu}$ of the periodic bias. Since the LS's lose their stability on increasing $\bar{\mu}$ the SR inhibition gradually becomes less relevant. A large kink density now gives rise to an enhancement of SR.

For the remainder of our investigation it is convenient to introduce the occupancy of the states $A_{1 \pm}$. We first introduce the total length of the segments of $U_{t}(x)$ that are positive (negative) at time $t$, which is considered as a parameter. This is given by $y^{+}(t)=m\left(B_{t}^{+}\right)\left[y^{-}(t)=m\left(B_{t}^{-}\right)\right]$, where $B_{t}^{+(-)}=\left\{x \in[0, L]: U_{t}(x)>0\left[U_{t}(x)<0\right]\right\}$, and $m$ is the Lebesgue measure. The occupancy $Y^{ \pm}$of the states $A_{1 \pm}$ is defined as

$$
Y^{ \pm}=\frac{\left\langle y^{ \pm}(t)\right\rangle}{L} .
$$

Moreover, we introduce the normalized averaged kink density $\rho$,

$$
\rho=\frac{\left\langle n_{k}(t)\right\rangle s_{0}}{L}=\frac{\left\langle\rho_{k}(t)\right\rangle}{\rho_{p}},
$$

where $n_{k}(t)$ is the number of kinks on a length $L, \rho_{k}$ is the corresponding density, and $\rho_{p}=1 / s_{0}$ is the maximum density of kinks allowed for a stable solution of Eqs. (1) and (2) which corresponds to a periodic soliton solution with period $2 s_{0}$.

When $\epsilon \neq 0$ the evolution of the instantaneous kink density $\rho_{k}$ becomes a random process. The birth and death of a pair of kinks is equivalent to the production (annihilation) of a 0-order LS (cavity soliton). This is achieved when the distance $s$ between the crossing pairs becomes bigger (smaller) than the critical size $s_{u n}$, the separation between the zeros of the real part of the unstable soliton solution which represents a saddle point [19]. Therefore not all the zeros $x_{k}$ identify unequivocally a kink and in the statistical count we must consider only kink pairs separated at least by $s_{\text {un }}$. After a transient time the system loses memory of the initial condition $\rho_{k}^{0}=\rho_{k}\left(t_{0}\right)$ and its momentum $\left\langle\rho_{k}(t) \mid \rho_{k}^{0}, t_{0}\right\rangle$ at equilibrium assumes the stationary value $\rho$ which depends only on $\epsilon$ once other parameters have been fixed. At equilibrium and for $\bar{\mu}=0, U(x, t)$ is evenly distributed among the two equivalent states \pm 1 , therefore $Y^{ \pm}=\left\langle y^{ \pm}\right\rangle=\frac{1}{2}$. In the weak noise limit kinks can be easily tracked and their stochastic dynamics followed in time. Their evolution is described by switchings between the equilibrium distances $s_{j}$ for short interaction distances and a diffusivelike motion for larger distances.

\section{Stochastic resonance}

Let us now consider the case with both $\epsilon \neq 0$ and $\bar{\mu} \neq 0$. The dynamics of an isolated kink undergoes a superposition of Brownian and periodic driven motion which is described by a Langevin equation $[9,23]$ for the kink position. Spatiotemporal synchronization in spatially extended systems is achieved through the depletion of the less stable state and is activated by the nucleation of small domains that grow and shrink due to the front motion. This is a particular feature introduced by spatial coupling of either a diffusive or a diffractive nature.

We remind the reader that spatial solitons and LS's exist in the 1D models (1) and (2), just above the threshold for the degenerate signal generation. As we have shown in Sec. II C the existence limit of LS's in the presence of bias depends on the parameter $\Gamma$. Our measurements of SR for different values of $\Gamma$ are therefore very sensitive to the magnitude of the periodic bias. This suggests to us that a characterization of SR needs to be carried out in the parameter space $(\Gamma, \bar{\mu})$. The range of $\bar{\mu}$ is $0 \leqslant \bar{\mu}<\mu_{t h}$, where $\mu_{t h}$ is given by Eq. (4), while $\Gamma$ is typically chosen in the interval $[0.2,5]$.

Both the finite integration time and the time discretization act as filters, introducing the cutoff frequencies $1 / T_{f}$ and $1 /(2 \Delta t)$, respectively, where $T_{f}$ is the total time of the simulation and $\Delta t$ is the time step. They also dictate that $\Delta t \ll T$ $\ll T_{f}$ for the period $T=2 \pi / \omega$ of the bias. For the numerical simulations we use a grid of typically 512 elements, an integration time $T_{f}=128 T$, with $T=10^{2}$, and a time step $\Delta t$ $=10^{-3}$. Other parameters are $a=0.5$ and $E=3$ which gives $\mu_{t h} \sim 1.0887$. These shall be considered fixed throughout the remainder of this paper. SR is observed on tuning the noise intensity $\epsilon$. For fixed values $\Gamma$ and $\bar{\mu}$, we run a series of simulations (with different noise realizations) and increase $\epsilon$ at each step. Among the many ways to measure SR we employ the SNR, defined as

$$
\theta=10 \log _{10}\left(\frac{\left[\Lambda(\Omega)-\Lambda_{N}(\Omega)\right]}{\Lambda_{N}(\Omega)}\right),
$$

where $\Lambda_{N}(\Omega)$ represents the spectral power of the background in the proximity of the signal peak. At our convenience we compare it with other SR quantifiers introduced below. Since the soliton width changes with $\Gamma$ [24], the length $L$ of the transverse field is chosen in such a way as to contain the same number of $s_{0}$. In our case $L=50 s_{0}$. However, we remark that a change in the spatial scale does not influence our numerical results as long as $1 \ll L / s_{0} \ll N$, which is imposed by the space discretization only.

Transitions between the stable states are rare at weak noise intensities and the SNR, which is initially low, rises on increasing $\epsilon$, and peaks at an optimal noise intensity $\epsilon_{S R}$ [see Fig. 5(a)]. For higher $\epsilon$ the transitions become less correlated and eventually the SNR dies out. Qualitatively similar behavior is observed in the parameter region considered here but with different values of $E$. A rich variety of behaviors is observed in the way SR occurs on varying $\Gamma$. On increasing $\Gamma$ the shape of the SNR curves changes continuously from broad to narrow while their maxima $\theta_{M}$ increase. To visualize this, we plot in Fig. 5 the curves corresponding to $\Gamma$ $=0.2$ (crosses), $\Gamma=1$ (empty squares), $\Gamma=5$ (triangles) for the driving amplitude $\bar{\mu}=0.1 \mu_{t h}\left(<\alpha_{0}\right)$. Note how the SNR spectra asymptotically $(\Gamma \rightarrow+\infty)$ approach the SNR curve obtained from numerical simulations of Eq. (2), which is represented by the dashed line in Fig. 5(a). This increase in 


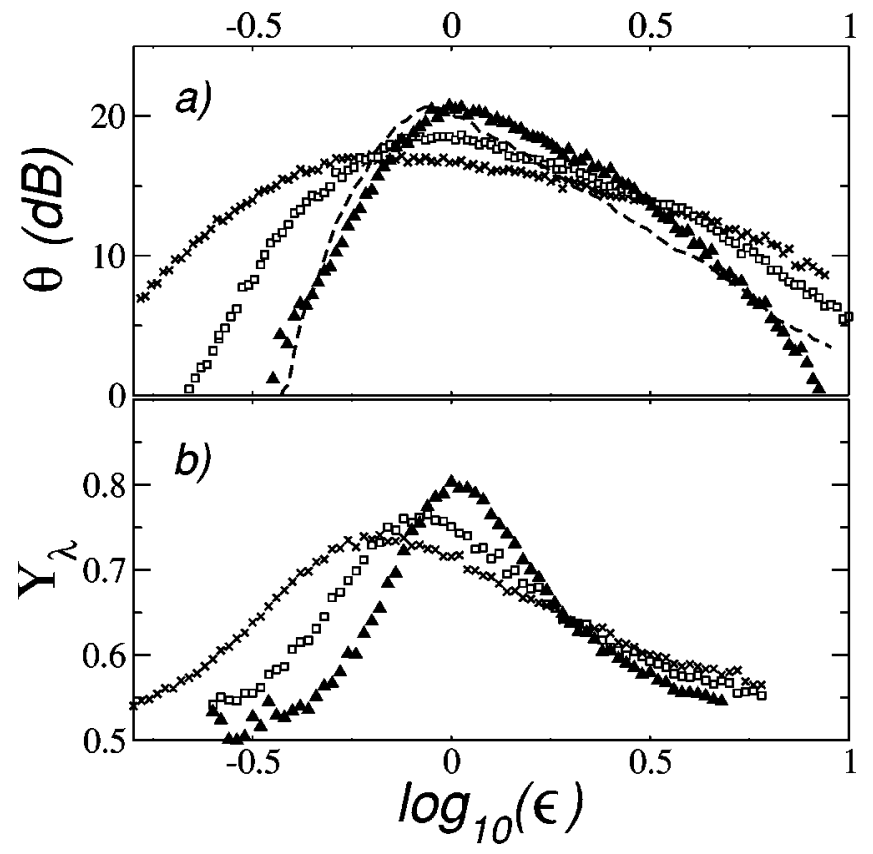

FIG. 5. (a) Signal-to-noise ratio curves for Eq. (1) when $\bar{\mu}$ $=0.1 \mu_{t h}$. Starting from the broadest curve: $\Gamma=0.2$ (crosses), $\Gamma$ $=1$ (squares), and $\Gamma=5$ (triangles). Dashed curve is obtained from numerical simulations of Eq. (2). b) Maximum occupancy $Y_{\lambda}$ for the same values $\Gamma=0.2$ (crosses), $\Gamma=1$ (squares), $\Gamma=5$ (triangles). Other parameters are $a=0.5, E=3$, and $\mu_{t h}=0.108866$.

the maximum $\theta_{M}$, which appears as an enhancement of SR, might resemble the AESR of Refs. [8,9]. However we stress that in our case enhancement of SR is not obtained by tuning the diffraction coefficient. This would in fact lead to a mere change of scale for the spatial coordinates. Note also that $\theta_{M}$ saturates to the asymptotic limit given by Eq. (2) rather than showing a maximum as in Ref. [8].

We now take a closer look at the dynamics of the field. The sequences shown in Fig. 6 represent the evolution of $u$ over eight periods of the driving for $\Gamma=0.2$ [panels $(\mathrm{a}-\mathrm{e})$ ] and $\Gamma=5$ [panels $(\mathrm{f}-\mathrm{j})$ ], respectively, and for increasing val-

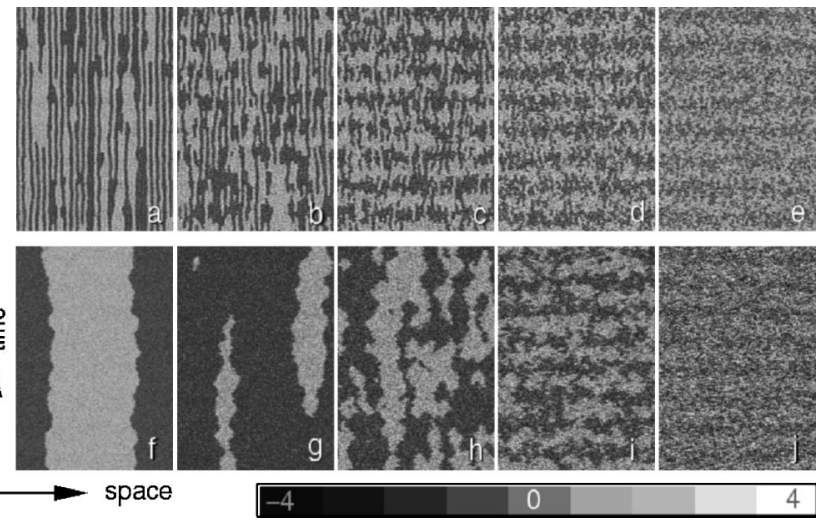

FIG. 6. Evolution of $u(x, t)$ for $\Gamma=0.2(\mathrm{a}-\mathrm{e})$ and $\Gamma=5(\mathrm{f}-\mathrm{j})$ for increasing values of $\epsilon$ : $\log _{10}(\epsilon)=-0.7(\mathrm{a}, \mathrm{f}),-0.45(\mathrm{~b}, \mathrm{~g}),-0.2$ (c,h), 0 (d,i), 0.25 (g,j), when $\bar{\mu}=0.1 \mu_{t h}$. Dark (light) regions represent positive (negative) values of $u$. Other parameters are as in Fig. 5.

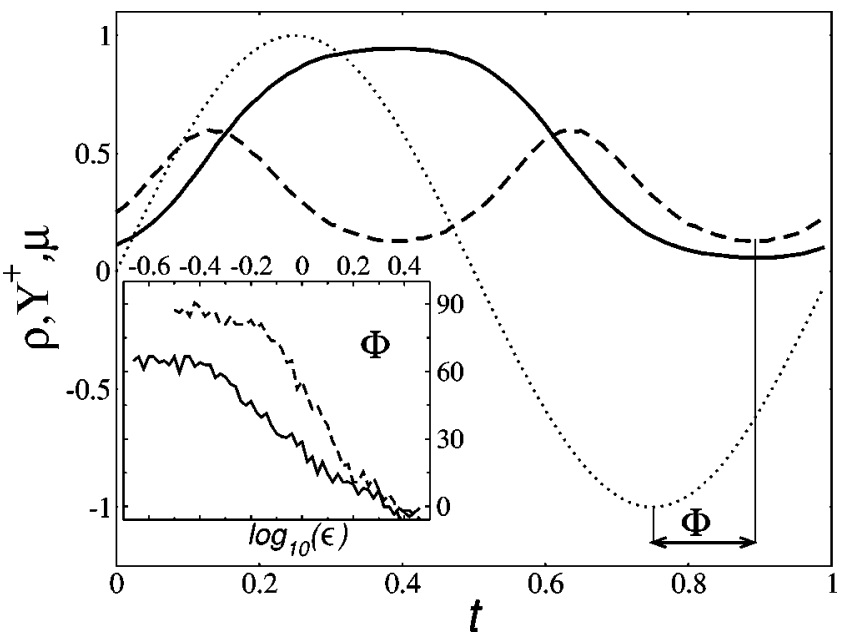

FIG. 7. Comparison between $\rho$ (dashed line), $Y^{+}$(solid line), and the periodic bias $\mu$ (dotted line), where $\bar{\mu}$ is set to 1 for convenience in the picture. The parameters are $\bar{\mu}=0.3 \mu_{t h}, \log _{10}(\epsilon)$ $=-0.2, \Gamma=0.2$. The inset shows the phase lag (degree) as function of $\log _{10}(\epsilon)$ for $\Gamma=0.2$ (solid line) and $\Gamma=5$ (dashed line). Other parameters are as in Fig. 3.

ues of $\epsilon$. From left to right we have $\log _{10}(\epsilon)=-0.7$ [panels $(\mathrm{a}, \mathrm{f})],-0.45$ [panels $(\mathrm{b}, \mathrm{g})],-0.2$ [panels $(\mathrm{c}, \mathrm{h})$ ], 0 [panels (d,i)], 0.25 [panels $(\mathrm{g}, \mathrm{j})$ ], when $\bar{\mu}=0.1 \mu_{t h}$. Dark (light) regions represent positive (negative) values of $u$.

We first analyze sequence $(\mathrm{a}-\mathrm{e})$ of Fig. 6 at $\Gamma=0.2$. We notice that a substantial kink density is already present for small $\epsilon$ [Fig. 6(a)] in accordance with ( $\left.i^{\prime}\right)$. LS's show up as stripes, the thinnest stripes corresponding to cavity solitons. Other LS's at larger distances $s_{j}(j>0)$ are less clearly identifiable as they have a shorter lifetime. The increasing number of birth-death events on increasing $\epsilon$ tends to synchronize with $\mu$ although the LS's are still visible even at resonance [Fig. 6(c)] causing a mixing between the two states, which prevents a full synchronization. In the last sequence $(\Gamma=0.2)$ of Figs. $6(\mathrm{f}-\mathrm{j})$, the kink population is initially negligible [Fig. 6(f,g)]. However here the synchronization is more effective at resonance [Fig. 6(i)]. This cannot be fully appreciated by looking at a short evolution of the field and needs to be proven by a statistical analysis that considers much longer simulations and quantifiers other than the SNR. For this reason we introduced the average occupancy $Y^{ \pm}$, and density $\rho$, that we shall now analyze.

The stochastic processes $y^{ \pm}$and $\rho_{k}$ are no longer stationary [25] in the presence of a periodic bias and their means $Y^{ \pm}$and $\rho$ become periodic functions of time $Y^{ \pm}(t)=Y^{ \pm}(t$ $+T)$ and $\rho(t)=\rho(t+T / 2)$, respectively. The curves $Y^{+}(t)$ (solid line) and $\rho(t)$ (dashed line), both obtained from numerical simulations and $\mu(t)$ (dotted line) are plotted in Fig. 7.

It is easy to see that the stationary points of $Y^{ \pm}$have to coincide with minima of $\rho$ whereas points where $Y^{+}=Y^{-}$ $=1 / 2\left(d^{2} Y^{+} / d t^{2}=0\right)$ correspond to maxima of $\rho$. In obtaining $Y^{ \pm}$and $\rho$ one has to take into account the phase lag in the spatiotemporal synchronization [26]. As a matter of fact the extrema $t_{s t}$ of $Y^{ \pm}$(minima of $\rho$ ) in the interval $(t, t$ 


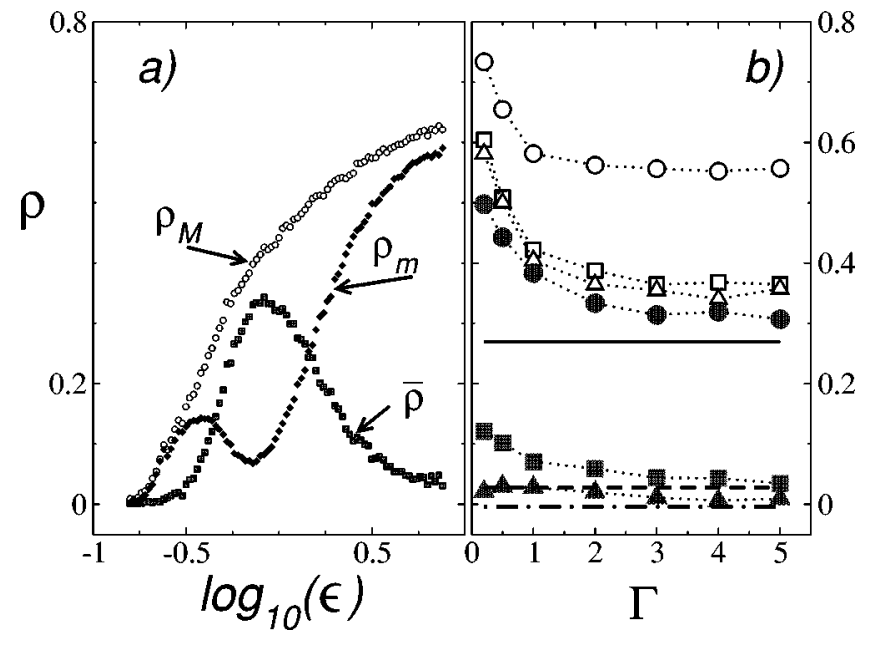

FIG. 8. (a) $\rho_{m}$ (diamonds), $\rho_{M}$ (empty circles), and $\bar{\rho}$ (squares) for $\bar{\mu}=0.25 \mu_{t h}$ and $\Gamma=1$. $\Gamma$. (b) Minimum and maximum defect densities at resonance: $\rho_{m}^{S R}$ (filled symbols) and $\rho_{M}^{S R}$ (empty symbols), respectively, for the values of bias amplitude $\bar{\mu}=0.15 \mu_{t h}$ (circles), $\bar{\mu}=0.25 \mu_{t h}$ (squares), and $\bar{\mu}=0.4 \mu_{t h}$ (triangles). Corresponding values of $\rho_{m}$ for Eq. (2) at $\bar{\mu}=0.15 \mu_{t h}$ (solid line), $\bar{\mu}$ $=0.25 \mu_{t h}$ (dashed line), $\bar{\mu}=0.4 \mu_{t h}$ (dotted-dashed line).

$+T]$ do not correspond to the extrema of $\mu$, which are $t_{s t}^{\prime}$ $=(2 n+1) T / 4 \quad(n=0,1,2, \ldots)$. Instead they are phase shifted by $\Phi(\epsilon)$, i.e., $t_{s t}=t_{s t}^{\prime}+\Phi$. It can be seen from the inset in Fig. 7 that the phase shift is not negligible especially for low noise levels.

The knowledge of one of $Y^{ \pm}$determines completely the other. Therefore the maximum occupancy $Y_{\lambda}$ of either $A_{1+}$ or $A_{1-}$ is achieved for $t=t_{s t}$ where $Y_{\lambda}=\left|Y^{ \pm}\left(t_{s t}\right)-1 / 2\right|$ $+1 / 2$, with $1 / 2 \leqslant Y_{\lambda} \leqslant 1$. Here the dependence on the parameter $\epsilon$ of the variables $\rho$ and $Y$ is implicitly assumed. A similar definition of occupancy which ignores the phase lag was given in Ref. [8]. Not surprisingly the maximum occupancy $Y_{\lambda}$ and SNR curves behave in a similar way, as is clear from Fig. 5, which compares the two quantities. As a matter of fact $Y_{\lambda}$ differs from the SNR because it only takes into account the signal power $\Lambda(\Omega)$ and not $\Lambda_{N}(\Omega)$. On increasing $\Gamma$ from $\Gamma=0.2$ (crosses) to $\Gamma=1$ (squares) to $\Gamma$ $=5$ (triangles), the curves $Y_{\lambda}(\epsilon)$ become narrower [Fig. 5(b)]. Furthermore their maximum increases and, as in Fig. 5(a), shifts to higher noise levels. Rather than thinking of it as an enhancement of SR on increasing $\Gamma$ we should rather consider this as a reduction in the inhibition caused by the locking of kinks. Although the interpretation of these results is supported by the properties (i)-(iii) and (i') and (ii') described earlier in Sec. II C, we need to look for a more quantitative proof of this picture.

We now consider the kink density which is itself a SR quantifier and in particular we focus on its minimum $\rho_{m}$ $=\rho\left(t_{s t}\right)$ and maximum $\rho_{M}=\rho\left(t_{s t}+T / 4\right)$.

Figure 8(a) shows the typical behavior of $\rho_{m}$ (diamonds), $\rho_{M}$ (circles), and $\bar{\rho}=\rho_{M}-\rho_{m}$ (squares) versus $\epsilon$. One notes that $\bar{\rho}$ also displays a resonance on tuning the noise level, at a value $\epsilon \sim \epsilon_{S R}$. However, the crucial quantity here is $\rho_{m}^{S R}$ $=\rho_{m}\left(\epsilon_{S R}\right)$, which represents the minimum kink density at resonance. Why this point is crucial is evident from the fact that the synchronization of $u$ requires the suppression of kinks. The most effective synchronization is the one where no kinks are present at $t_{s t}$. This is equivalent to $Y_{\lambda}\left(\epsilon_{S R}\right)$ $=1, \rho_{m}^{S R}=0$. Typically, however, $Y_{\lambda}\left(\epsilon_{S R}\right)<1$ and $\rho_{m}^{S R}>0$. Figure 8(b) shows that $\rho_{m}^{S R}$ decreases monotonically on increasing $\Gamma$ for $\bar{\mu}=0.15 \mu_{t h}$ (filled circles) so that $\rho_{m}^{S R}$ is much higher than zero for low values of $\Gamma$. Here the motion of the kinks is inhibited by the fact that they are so close to each other that they form metastable states. These kinks are not isolated since their average distance is $1 / \rho_{m}^{S R} \sim 2$ in $s_{0}$ units. In Fig. 8(b) we also plot the value of $\rho_{m}^{S R}$ for Eq. (2) (solid line) which is approached asymptotically by Eq. (1) as $\Gamma$ $\rightarrow \infty$. At $\Gamma=5$ the average distance between kinks is $1 / \rho_{m}^{S R}$ $\sim 4$. The spatial oscillations in this case are critically damped and are not able to give rise to metastable states of order higher than 1 . Hence kinks can already be considered as independent at this average distance.

As we pointed out earlier, solitons are stable against contraction induced by the bias when $\bar{\mu}<\alpha_{0}$. This is the weakest condition in order to maintain a certain number of stable kinks in the deterministic case and in the presence of bias. However, kinks can annihilate due to the presence of stochastic fluctuations that can induce a collision between a kink and an antikink which results in their annihilation. In this case the kink density is determined by the ratio between $\mathcal{E}_{a}$ and $\mathcal{E}_{d}$, as pointed out in (ii'). The existence limit of the solitons does not have a great influence on the kink density for small $\bar{\mu}$. As a matter of fact, $\rho_{m}^{S R}(\Gamma)$ is monotonic while $\alpha_{0}(\Gamma)$ [see Fig. 3(b)] displays a maximum for $\Gamma_{\alpha_{0}} \sim 0.5$. On reducing $\Gamma$ from the value $\Gamma_{\alpha_{0}}$ the kink density $\rho_{m}^{S R}$ increases though solitons become "less stable." The key point here is the relation between the two important quantities $\mathcal{E}_{a}$ and $\mathcal{E}_{d}$. When $\mathcal{E}_{a} \sim \mathcal{E}_{d}$ a larger kink density than in the case $\mathcal{E}_{a}>\mathcal{E}_{d}$ is formed.

This holds until $\bar{\mu}<\alpha_{0}$, since close to $\alpha_{0}$ the deterministic dynamics becomes more relevant. Evidently, $\rho_{m}^{S R}$ decreases on increasing $\bar{\mu}$, because collisions become more likely, but it decreases at different rates for different values of $\Gamma$. Note that when a temporary equilibrium between the two phases is established $\rho$ has a maximum, $\rho=\rho_{M}$ (see Fig. 8). For low values of $\Gamma$ and at any value of the bias amplitude, the kink density $\rho_{M}$ is large and decreases monotonically on increasing $\Gamma$ [empty symbols in Fig. 8(b)]. It should be noted that large $\rho_{M}$ results in a more rapid annihilation of the kinks at $t_{s t}$, when $\bar{\mu}>\alpha_{0}$. As a matter of fact, the closer the kinks are the easier it is for the bias to erase them and, indeed, large $\rho_{M}$ means closer interaction distances. Hence, on increasing $\bar{\mu}, \rho_{m}^{S R}$ decreases more rapidly at small $\Gamma$ than at large $\Gamma$. This is shown by the curves $\bar{\mu}=0.25 \mu_{t h}$ (filled squares) and $\bar{\mu}=0.4 \mu_{t h}$ (filled triangles) in Fig. 8(b). In the last curve one observes in particular a nonmonotonic behavior which is the signature of a substantial change in the SR curves, as we shall see later. Dashed and dotted-dashed lines in Fig. 8(b) are again the values of $\rho_{m}^{S R}$ for Eq. (2) which plays the role of asymptotic values of the curves $\rho_{m}^{S R}$, for 


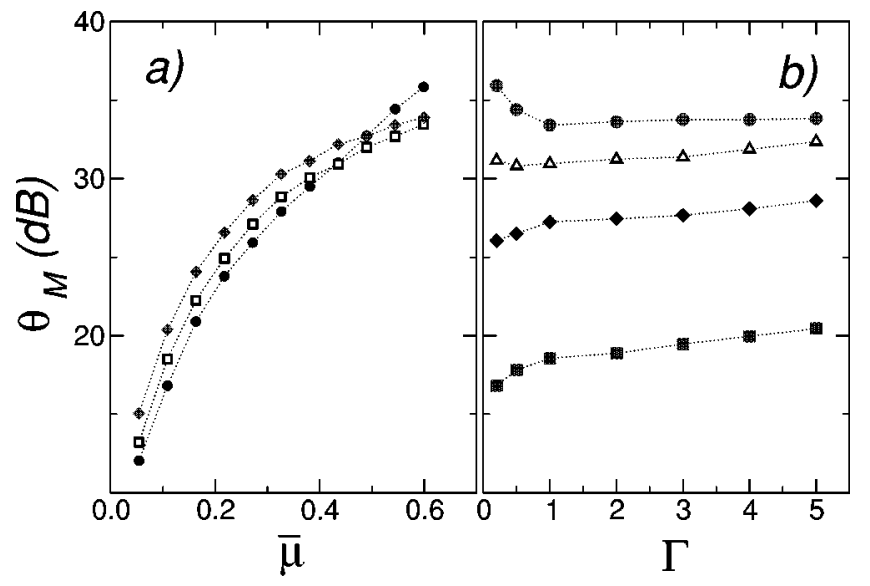

FIG. 9. (a) Maximum $\theta_{M}$ of the SNR against $\bar{\mu}: \Gamma=0.2$ (circles), $\Gamma=1$ (squares), and $\Gamma=5$ (diamonds). (b) Curves $\theta_{M}$ against $\Gamma: \bar{\mu}=0.1 \mu_{t h}$ (squares), $\bar{\mu}=0.25 \mu_{t h}$ (filled diamond), $\bar{\mu}$ $=0.4 \mu_{t h}$ (triangles), and $\bar{\mu}=0.55 \mu_{t h}$ (filled circles).

$\Gamma \rightarrow+\infty$. For comparison we also plot the maximum kink density $\rho_{M}^{S R}=\rho_{M}\left(\epsilon_{S R}\right)$ at resonance for the same values of the bias amplitude $\bar{\mu}=0.15 \mu_{t h}$ (empty circles), $\bar{\mu}=0.25 \mu_{\text {th }}$ (empty squares), and $\bar{\mu}=0.4 \mu_{t h}$ (empty triangles).

The onset of signal amplification decreases on reducing $\Gamma$ due to the increasing kink density. Therefore the SNR and occupancy curves, at low noise intensities, increase in width as $\Gamma$ decreases. On increasing $\bar{\mu}$, the onset of SR decreases even further when $\Gamma$ is small. This phenomenon is easily explained by referring to the kink density. For weak noise levels the birth-death events become so rare that $\rho$ may be thought of as a stationary process $\left(\rho_{m} \cong \rho_{M}\right)$. In this circumstance the signal power at the driving frequency is overwhelmingly due to front motion. Typically, on reducing $\epsilon$ the kink density becomes so small that this effect is hardly observable. However, when $\mathcal{E}_{a} \sim \mathcal{E}_{d}(\Gamma \sim 0.2), \rho$ is significantly greater than zero even for very low noise intensities. Here, the contribution given by the driven fronts to the spectral component $\Omega / 2 \pi$ becomes relevant and indeed leads to a lowering of the onset of SR. When $\mathcal{E}_{a} \gg \mathcal{E}_{d}$, on the other hand, the kink density $\rho$ tends to zero very rapidly for $\epsilon \rightarrow 0$.

We now focus on the behavior of the SNR, $\theta$, on increasing $\bar{\mu}$. We consider here the maximum $\theta_{M}$ of $\theta$. Plots of $\theta_{M}$ versus $\bar{\mu}$ and $\Gamma$ are given in Figs. 9(a) and 9(b), respectively. Figure 9(a) shows the growth of $\theta_{M}(\bar{\mu})$ for the values $\Gamma$ $=0.2$ (circles), $\Gamma=1$ (squares), and $\Gamma=5$ (diamonds). The monotonic growth of $\theta_{M}$ is obviously due to the increase of the bias amplitude. What is interesting to observe, however, is the relative growth of $\theta_{M}$ for different values of $\Gamma$. One notices that the distance between the curves tends to decrease on increasing $\bar{\mu}$. In particular $\theta_{M}$ grows faster for low $\Gamma$ (circles). This is significant in that the inhibition of SR induced by the presence of cavity solitons decreases for larger $\bar{\mu}$. More importantly a large density $\rho_{M}(S R)$ eventually helps a more effective synchronization leading to an enhancement of SR.
To appreciate this phenomenon more easily we plot in Fig. 9(b) the curves $\theta_{M}(\Gamma)$ for $\bar{\mu}=0.1 \mu_{t h}$ (squares), $\bar{\mu}$ $=0.25 \mu_{t h}$ (filled diamond), $\bar{\mu}=0.4 \mu_{t h}$ (triangles), and $\bar{\mu}$ $=0.55 \mu_{t h}$ (filled circles). The transition from inhibition to enhancement of SR is explained by the reduced effectiveness of the locking of kinks. This is more apparent for small $\Gamma$ where a larger kink density causes the inhibition of SR when $\bar{\mu}<\alpha_{0}$ but, on the other hand, favors the quick annihilation of kinks when $\bar{\mu}>\alpha_{0}$. As a matter of fact kink pairs with a shorter interaction distance are more likely to annihilate. Consequently the curves $\theta_{M}(\Gamma)$ [Fig. 9(b)] that increase monotonically for $\bar{\mu}=0.1 \mu_{t h}$ change character, eventually becoming decreasing functions of $\Gamma$.

\section{2D CASE}

In this section we examine the occurrence of SR in the two-dimensional DRDOPO model (1). We have seen that in one dimension, LS's and cavity solitons emerge at the threshold $E_{t h}$ for parametric down-conversion and are stabilized due to a balance between diffraction and nonlinearity. In two dimensions the onset of the existence of stable structures does not coincide with the degenerate signal threshold because of curvature phenomena that now have to be taken into account. For instance, at $\Gamma=1$, the threshold $E_{0}$ for the 0 -order cavity soliton is at $E \cong 2.2$ while the threshold for the degenerate signal generation is $E_{t h}=1$. Higher order LS's appear for increasingly higher pump values, in such a way that their thresholds $E_{j}$ obey $E_{j}<E_{j+1}$ [13]. This is similar to the $1 \mathrm{D}$ case in the presence of a spatially uniform constant bias $\mu$. Even there the thresholds $E_{j}$ are found on increasing $E$ when $0<|\mu|<\mu_{t h}$. This can be seen from Fig. 1 upon moving upward along a vertical line. Earlier in Sec. II A we pointed out that it is possible to reduce consistently the thresholds $E_{j}$ by reducing $\Gamma$ [14]. These, however, remain above the value $E_{t h}$.

The dynamics of the transverse degenerate signal field shows phase-ordering-like kinetics typical of nonequilibrium systems, where the order parameter is not conserved [27]. A domain of one phase shrinks and eventually disappears in the opposite phase for $E_{t h}<E<E_{0}$. Instead for $E_{j}<E<E_{j+1}$, the shrinking of a domain, which can be accompanied by the disappearance of any domain embedded in it, leads eventually to the stabilization of a LS of circular shape with diameter $s_{j}$.

It is easy to see that quantities such as the power spectrum $\Lambda$ can be immediately extended to the $2 \mathrm{D}$ case. From this we can obtain the SNR which gives information about the SR phenomenon. With the arguments employed in the preceding section it is possible to show that SR presents qualitatively the same properties as in one dimension. We use a grid of $128 \times 128$ elements, and a driving amplitude $\bar{\mu}=0.15$. From the numerical side the extra spatial degree of freedom results in a significant increase in the integration time. The length of our simulations is now 50T. Other parameters are as in the preceding section. Note that the addition of a spatial degree of freedom does not affect $\mu_{t h}$ and the homogeneous solutions. 
a)

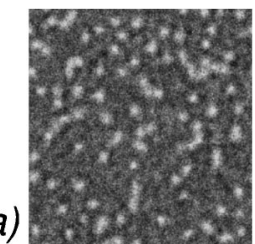

b)

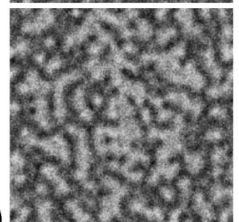

c)

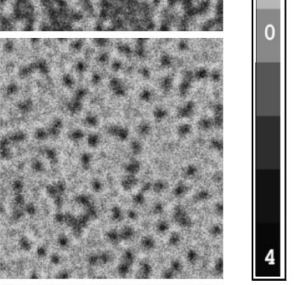

d)

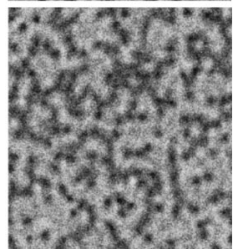

e)

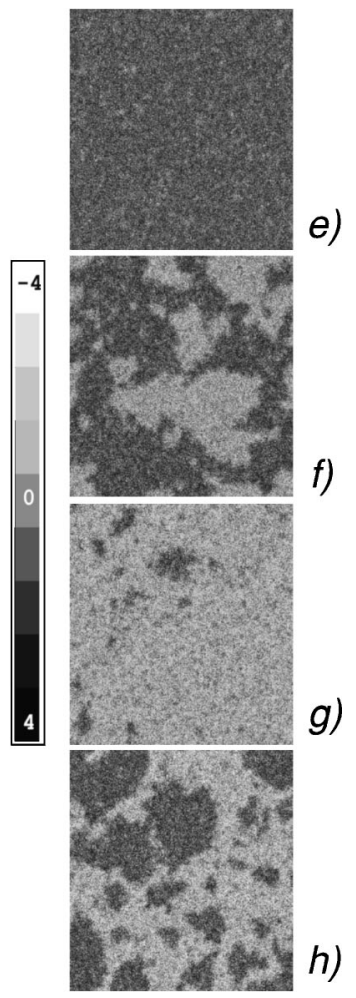

FIG. 10. From top to bottom, the sequences show the evolution of $u(\vec{x}, t)$ during one period of $\mu(t)$ taken at intervals of $T / 4$. (a) Right: $\Gamma=0.1$. (b) Left: $\Gamma=5$. Other parameters are $E=3$ and $\bar{\mu}$ $=0.15 \mu_{t h}$.

We first analyze the near field. In Fig. 10, the two sequences $(\mathrm{a}-\mathrm{d})$ and $(\mathrm{e}-\mathrm{h})$ show the evolution of $u$ during one period $T$ of $\mu(t)$. For $\Gamma=0.1$ (right) and $\Gamma=5$ (left) we have $t=t^{\prime} \quad$ [panels (a,e)], $t=t^{\prime}+T / 4$ [panels (b,f)], $t=t^{\prime}+T / 2$ [panels $(\mathrm{c}, \mathrm{g})$ ] , and $t=t^{\prime}+3 T / 4$ [panels $(\mathrm{d}, \mathrm{h})$ ], where $t^{\prime}$ is an appropriate time. At $t=t^{\prime}$ [panels $\left.(\mathrm{a}, \mathrm{e})\right], u$ is at the point of maximum occupancy of the state $A_{1+}$ (first row). Subsequently the periodic driving helps domains of the minority phase to grow and in panels (b,f) $u$ is equally distributed between the two phases. At this point the negative phase starts to be preferred and in panels $(\mathrm{c}, \mathrm{g}) u$ reaches the point of maximum occupancy of the state $A_{1-}$. Again in panels $(\mathrm{d}, \mathrm{h})$ we have a temporary balance between the two phases and subsequently the entire evolution periodically repeats in time. In the left sequence $(\Gamma=0.1)$ of Fig. 10 the bright and dark spots, clearly visible in (a) and (c), are cavity solitons in two dimensions. Their presence is an obstacle to the synchronization process. This takes place more effectively for $\Gamma$ $=5$ (right sequence) where at the point of maximum occupancy of $A_{1 \pm}$ the transverse field consists almost entirely in one phase [see panels $(\mathrm{e}, \mathrm{g})]$. The SNR curves shown in Fig. 11 for $\Gamma=0.1$ (circles), $\Gamma=1$ (squares), and $\Gamma=5$ (triangles) display the same qualitative behavior as Fig. 5. The maximum $\theta$ of the SNR increases with $\Gamma$ and the curves become narrower. Therefore the inhibition of SR decreases on increasing $\Gamma$. However, on increasing $\bar{\mu}$ the maxima increase at different rates for different $\Gamma$ and eventually an enhancement of SR is achieved for low $\Gamma$ as in one dimension. These

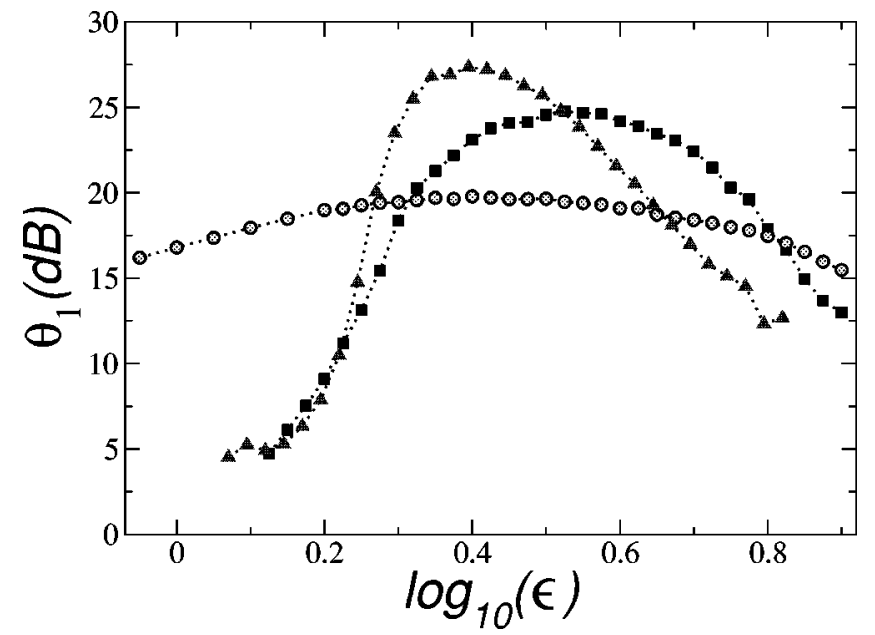

FIG. 11. SNR curves for Eq. (1) in two dimensions on varying $\Gamma$ at the bias amplitude $\bar{\mu}=0.15 \mu_{t h}$. Starting from the broadest curve: $\Gamma=0.1$ (circles), $\Gamma=1$ (squares), and $\Gamma=5$ (triangles).

results support the idea that SR in two dimensions presents qualitatively the same features shown by the $1 \mathrm{D}$ dynamics.

\section{CONCLUSIONS}

We have shown that SR, seen as a synchronization of noise-induced transitions between two states in the transverse field of OPO systems, can be enhanced (inhibited) on increasing the kink density for large (small) driving amplitudes. We have characterized SR by tuning the pump cavity finesse, i.e., the parameter $\Gamma$. This is seen to affect the LS profile, modifying the ratio between activation and deactivation amplitudes for the creation and annihilation of solitons. Eventually this results in a variation of the average kink density. Hence, we demonstrated that locking kinks at low driving amplitudes inhibit SR and this inhibition increases with the kink density. We have also shown that, at larger driving amplitudes, in the regime where LS's cease to be stable, a large density of kinks is instead beneficial to an effective synchronization. Moreover we presented a suitable generalization of the SR effect to the two-dimensional case where the validity of our interpretation holds true. The described phenomena are universal once two equivalent homogeneous states and DW's leading to locked solitons are present. For this reason we believe that the implications of our investigation are not limited to nonlinear optics but can be generalized to other fields of science including fluid dynamics, chemical oscillations, and solid state physics.

Finally, we note that our SR corresponds to synchronization of the order parameter. A complementary interpretation considering the kinks as particlelike objects is possible and will be presented elsewhere [28].

\section{ACKNOWLEDGMENTS}

We acknowledge support from SGI, EPSRC (Grant Nos. GR/M19727, GR/M31880, and GR/R04096) the EC (Contract No. IST-2000-26019 QUANTIM) and SHEFC (grants VISION and VIDEOS). 
[1] R. Benzi, G. Parisi, A. Sutera, and A. Vulpiani, Tellus 34, 10 (1982).

[2] L. Gammaitoni, P. Hängi, P. Jung, and F. Marchesoni, Rev. Mod. Phys. 70, 223 (1998).

[3] J. García-Ojalvo and J. M. Sancho, Noise in Spatially Extended Systems (Springer-Verlag, New York, 1999).

[4] P. Jung and G. Mayer-Kress, Phys. Rev. Lett. 74, 2130 (1995).

[5] K. Wiesenfeld, Phys. Rev. A 44, 3543 (1991); P. Jung, U. Behn, E. Pantazelou, and F. Moss, ibid. 46, R1709 (1992).

[6] P. Jung, U. Behn, E. Pantazelou, and F. Moss, Phys. Rev. A 46, R1709 (1992).

[7] F. Castelpoggi and H.S. Wio, Europhys. Lett. 38, 91 (1997); B. Von Haeften et al., Phys. Rev. Lett. 84, 404 (2000).

[8] J.F. Lindner et al., Phys. Rev. Lett. 75, 3 (1995).

[9] F. Marchesoni, L. Gammaitoni, and A.R. Bulsara, Phys. Rev. Lett. 76, 2609 (1996).

[10] M. Löcher, G.A. Johnson, and E.R. Hunt, Phys. Rev. Lett. 77, 4698 (1996).

[11] J. Opt. Soc. Am. B 16 (1999), special issue on Optical Parametric Devices and Processes.

[12] G.-L. Oppo, M. Brambilla, and L. Lugiato, Phys. Rev. A 49, 2028 (1994); G.J. de Valcarcel, K. Staliunas, E. Roldan, and V.J. Sanchez-Morchillo, ibid. 54, 1609 (1996); S. Longhi, ibid. 53, 4488 (1995); A. Gatti and L.A. Lugiato, ibid. 52, 1675 (1995); A. Gatti, H. Wiedemann, L.A. Lugiato, I. Marzoli, G.-L. Oppo, and S.M. Barnett, ibid. 56, 877 (1997); I. Marzoli, A. Gatti, and L. Lugiato, Phys. Rev. Lett. 78, 2092 (1997).

[13] G.-L. Oppo, A.J. Scroggie, and W.J. Firth, Phys. Rev. E 63, 066209 (2001).
[14] G.-L. Oppo, A.J. Scroggie, S. Sinclair, and M. Brambilla, J. Mod. Opt. 47, 2005 (2000).

[15] M. Vaupel, A. Maitre, and C. Fabre, Phys. Rev. Lett. 83, 5278 (1999).

[16] T. Alarcón, A. Pérez-Madrid, and J.M. Rubí, Phys. Rev. E 57, 4979 (1998).

[17] We prefer to label the down-converted beam in the OPO cavity as "degenerate signal" to distinguish it from the more general meaning of "signal" employed, for example, in the SNR measures.

[18] P. Coullet, C. Elphick, and D. Repaux, Phys. Rev. Lett. 58, 431 (1987).

[19] I. Rabbiosi, A.J. Scroggie, and G.-L. Oppo, Phys. Rev. Lett. 89, 254102 (2002).

[20] M. San Miguel and R. Toral, Stochastic Effects in Nonlinear Systems, Proc. IEEE, 85, 1 (1997).

[21] W.J. Firth and A.J. Scroggie, Phys. Rev. Lett. 76, 1623 (1996).

[22] T. Maggipinto, M. Brambilla, G.K. Harkness, and W.J. Firth, Phys. Rev. E 62, 8726 (2000).

[23] F. Marchesoni, Phys. Lett. A 115, 29 (1986); E. Joergensen, V.P. Koshelets, R. Monaco, J. Mygind, M.R. Samuelsen, and M. Salerno, Phys. Rev. Lett. 49, 1093 (1982).

[24] I. Rabbiosi, A.J. Scroggie, and G.-L. Oppo, Eur. Phys. J. D 22, 453 (2003).

[25] N. G. Van Kampen, Stochastic Processes in Physics and Chemistry (North-Holland, Amsterdam, 1992).

[26] B. McNamara and K. Wiesenfeld, Phys. Rev. A 39, 4854 (1989).

[27] A.J. Bray, Adv. Phys. 43, 357 (1994).

[28] I. Rabbiosi and G.-L. Oppo (unpublished). 